\title{
Publisher's Note: Equivalence between non-Markovian and Markovian Dynamics in Epidemic Spreading Processes \\ [Phys. Rev. Lett. 118, 128301 (2017)]
}

Michele Starnini, James P. Gleeson, and Marián Boguñá

Q (Received 27 July 2020; published 7 August 2020)

DOI: 10.1103/PhysRevLett.125.069902

This paper was published online on March 24, 2017 with an error in Eq. (12).

Equation (12) should read as

$$
\lambda_{\text {app }}=\frac{\hat{\psi}_{I}(2 \tilde{\delta})\left[1-\hat{\psi}_{I}(\tilde{\delta})+\langle k\rangle \hat{\psi}_{I}(\tilde{\delta})\right]-\langle k\rangle \hat{\psi}_{I}(\tilde{\delta})}{\hat{\Psi}_{I}(2 \tilde{\delta})\left[1-\hat{\psi}_{I}(\tilde{\delta})+\langle k\rangle \hat{\psi}_{I}(\tilde{\delta})\right]-\langle k\rangle \hat{\Psi}_{I}(\tilde{\delta})} .
$$

The equation has been corrected as of July 29, 2020. The equation is incorrect in the printed version of the journal. 